\title{
Characteristics of Telemetry Interference with Pacemakers Caused by Digital Media Players
}

\author{
ASHOK J. SHAH, M.D., * JOSEPH D. BRUNETT, PH.D., ${ }^{*}$ JAY P. THAKER,†
}

MEHUL B. PATEL, M.D., ${ }^{*}$ VALDIS V. LIEPA, PH.D., ${ }^{*}$ KRIT JONGNARANGSIN, M.D.,†

and RANJAN K. THAKUR, M.D.‡

From the *Thoracic and Cardiovascular Institute, Sparrow Health System, Michigan State University, Lansing,

Michigan; +Department of Electrical Engineering and Computer Science; and ‡Division of Cardiovascular Medicine,

University of Michigan, Ann Arbor, Michigan

Background: Contemporary implantable heart rhythm devices communicate multiple complex data simultaneously using radiofrequency telemetry. Interference in communication can expose them to the risk of potential corruption, leading to adverse clinical consequences.

Methods $\&$ Results: We studied the characteristics of interference with uplink (real time intracardiac electrograms, marker channel, and stored histograms) and downlink (attempt to program a change in the lower rate limit, the pacing mode, and the ventricular lead configuration) data transmission between the wand and the pacemaker caused by digital media players (iPods-Photo and 3G) in 50 patients. We also measured and characterized worst-case magnetic field emissions (MFE) from the wand $(\leq 0.4 \mu T)$, pacemaker $(\leq 0.004 \mu \mathrm{T})$, and iPod $(\leq 0.05 \mu \mathrm{T})$ during telemetry to understand the modulation techniques and safety protocols employed during data transmission. Telemetry interference (TI) manifested as highfrequency spikes (24.4\%), blanking (17.7\%) and interruption $(22.2 \%)$, or delay $(17.6 \%)$ in transmission with warning on programmer's screen. TI occurred in $25.6 \%$ of patients when the iPod was "on" and in $13 \%$ even with the iPod turned "off." There were no inaccuracies in downlinked data when the downlink communication was successful. Wanded telemetry utilizes low-frequency (30-300 kHz) radiowaves and simple digital modulation techniques at relatively slow rates for "sequential" data transmission protected by a continuous "handshake." Emissions from iPods in that range interrupt the telemetry link but are too weak to cause pacemaker malfunction through corruption of vital data.

Conclusion: Low-power MFE from iPods can produce interference with establishment and maintenance of a telemetry link and can cause TI with transmission of real time data, but because of continuous check protocols, do not corrupt the stored and vital downlink data. (PACE 2010; 33:712-720)

\section{telemetry interference, implantable pacemakers, digital media players}

\section{Introduction}

Telemetry interference (TI) is a poorly understood type of radiofrequency (RF) electromagnetic interference with implantable heart rhythm devices (IHRD), such as pacemakers, implantable cardiac defibrillators (ICDs), and implantable loop recorders (ILRs). TI has, hitherto, been considered clinically inconsequential. However, media players have been shown to cause TI with pacemakers and its interference with ILRs is recognized to have important clinical implications, impacting the utility of this innovative diagnostic tool. ${ }^{1-3}$ Chronic follow-up of IHRD patients using

Conflict of interest: None.

Address for reprints: Ranjan K Thakur, M.D., M.P.H., Thoracic and Cardiovascular Institute, 405 West Greenlawn, Suite 400, Lansing, MI 48910. Fax: 517-483-7568; e-mail: thakur@msu.edu

Received May 27, 2009; revised October 18, 2009; accepted November 12, 2009.

doi: 10.1111/j.1540-8159.2009.02672.x remote monitoring systems (RMS) is increasing in importance. Home-based monitoring systems for IHRDs utilize either remote or wanded (inductive) telemetry via a wireless communication link. Inductive telemetry (IT) using a wand communicates over a distance of few centimeters while remote telemetry has a range of a few meters. ${ }^{4}$ Short- and long-range wireless communication is now indispensable in both the domestic and the industrial arenas. RF identification (RFID) devices are now regularly used for tracking equipment in hospitals and books in libraries, for instance, and these RFID readers have been shown to cause clinically significant interference in pacemakers and ICDs, not to mention potentially hazardous electromagnetic interference with medical equipment in an intensive care unit. ${ }^{5-7}$ Recently, Halperin et al. described short-range cyber attacks using an inexpensive software radio to intercept and alter data from an ICD, raising concerns about privacy and safety issues related to wireless RF communication in IHRDs. ${ }^{8}$ Security and interference with wireless IHRDs is foreseen as a major upcoming challenge.

(C) 2010, The Authors. Journal compilation (C)2010 Wiley Periodicals, Inc. 
Understanding the types of RF telemetry communication used by pacemakers and troubleshooting telemetric problems is imperative, especially for safe and effective use of wireless monitoring of IHRDs. Since TI during wanded telemetry has been reported with media players, we used this model to study the characteristics of this interference to develop a better understanding of it.

\section{Methods}

This prospective, non-blinded study was performed on patients in the hospital or in an outpatient pacemaker clinic. The study was approved by the institutional review board and followed Good Clinical Practices guidelines, including the use of standard operating procedures. Written informed consent was obtained from all patients. Two types of media players were tested: Third Generation (3G) and Photo iPods. Both devices tested were manufactured prior to December 2005. Fifty consecutive patients who consented to the study were included; these patients had pacemakers manufactured by Boston Scientific, Medronic Inc., or St. Jude Medical.

Testing was performed on patients sitting upright at $90^{\circ}$ in the pacemaker clinic and supine at $30^{\circ}$ in hospital, with the pacemaker programmed to the patient's usual settings. If the patient with DDD(R) pacemaker was found to be in atrial fibrillation at the inception of the test, pacemaker mode was changed to VVI(R), ad-hoc.

\section{Protocol}

Continuous single-lead electrocardiographic (ECG) monitoring was established by connecting the patient to the programmer's ECG channel. After the pacemaker settings were printed at baseline, the histograms and event counters were reset. While the ECG and real time data were continuously printed through the test period, patient was exposed to the iPods (Photo and 3G) one at a time. The iPod was held within 2 inches of the pacemaker-wand interface. During the period of iPod exposure, intracardiac electrogram (EGM) (real time), marker channel (real time), and histogram (stored) data were collected from the pacemaker. Three commands for pacemaker reprogramming were delivered sequentially: (1) the lower rate limit was increased to 90-100 beats per minute, (2) pacing mode was programmed to $\operatorname{VVI}(\mathrm{R})$, if it was $\mathrm{DDD}(\mathrm{R})$ at baseline, and SOO, if it was SSI(R) at baseline, and (3) ventricular lead configuration was programmed from bipolar to unipolar or vice versa. After completing the test, the iPod was moved away and pre-test settings were restored.

\section{Classification}

Uplink Telemetry

Data (intracardiac EGMs, marker channel, and histograms) collected from the pacemaker constituted uplink telemetry. Three types of uplink TI were observed: (1) TI with intracardiac EGMs was observed as high-frequency spikes or blanking on the EGM channel during the test without corresponding interference on the ECG channel, (2) TI with marker channel was observed as highfrequency annotations or blanking of the marker channel during the test without corresponding interference on the ECG channel, and (3) TI with stored histogram data retrieved during the test period was observed as unusually high-rate bars without high rates on the ECG channel or the data could not be retrieved until the iPod was moved away from the wand-pacemaker interface. While testing for interference with real time uplink data (intracardiac EGMs and marker channel), if high frequencies and blanking happened to occur sequentially, we counted them as two observations per test.

\section{Downlink Telemetry}

Commands (change in the lower rate limit, the pacing mode, and the ventricular lead configuration) delivered from the programmer (via wand) to pacemaker constituted downlink telemetry. Three types of observations represented TI with downlink telemetry data: (1) downlink data transmission was delayed due to intermittent functional delinking at the wand-pacemaker interface manifested by pop ups but the data were eventually transmitted without inaccuracy, (2) downlink data transmission was interrupted until the iPod was moved away from the wand-pacemaker interface, and (3) downlink data transmission was inaccurate.

\section{Magnetic Field Emissions Testing}

\section{iPod Testing}

The iPod models, 3G and Photo, were evaluated for magnetic field emissions (MFE) in the frequency range of $10-500 \mathrm{kHz}$. The test setup was placed in the center of a 3-m Faraday cage and consisted of a sub-resonant shielded loop sensor (University of Michigan, DC-20 MHz operating range), high-impedance amplifier, and Agilent 8593E Spectrum Analyzer (SA; Agilent Technologies, Palo Alto, CA, USA). The loop sensor was fixed $1 \mathrm{~cm}$ from the front of each iPod ("On" and "Off" modes) and the front surface was scanned through all three axes to determine peak magnetic field emissions in a $10-\mathrm{kHz}$ bandwidth. At each measurement location, the sensor orientation was optimized to find the highest magnetic field 
strength. Emissions were then recorded on the SA and transferred to a personal computer for analysis. Emissions were observed with a similar magnitude across the frequency range of interest.

\section{Wand and Pacemaker Testing}

Close proximity MFE of three wandpacemaker pairs was evaluated over the frequency range of $10 \mathrm{kHz}-3 \mathrm{MHz}$ as above. We tested wands with the programmer model numbers 3120 (Boston Scientific Corporation, Natick, MA, USA-BSC), 2,090 W (Medtronic, Inc., Minneapolis, MN, USA-MDT) and 3650 (St. Jude Medical, Inc., St. Paul, MN, USA-SJM).

\section{Data Collection and Statistical Analysis}

Standardized data collection forms were used to collect information on demographics, implan- tation details, pacemaker lead(s) and generator, pacemaker parameters, and test results. All data were maintained in a Microsoft Excel database and analyzed using SPSS statistical software (SPSS, Inc., Chicago, IL, USA).

\section{Clinical Testing}

The study population consisted of 50 patients (25 men, mean age $70.3 \pm 6.9$ years). Amongst them, 24 patients had BSC devices, 11 patients had MDT devices, and 15 had SJM devices. We recorded 1,234 observations for 1,200 tests performed (Figs. 1 and 2). TI manifested as highfrequency spikes $(24.4 \%)$, blanking $(17.7 \%)$, and interruption $(22.2 \%)$ or delay $(17.6 \%)$ in transmission with warning on programmer's screen.

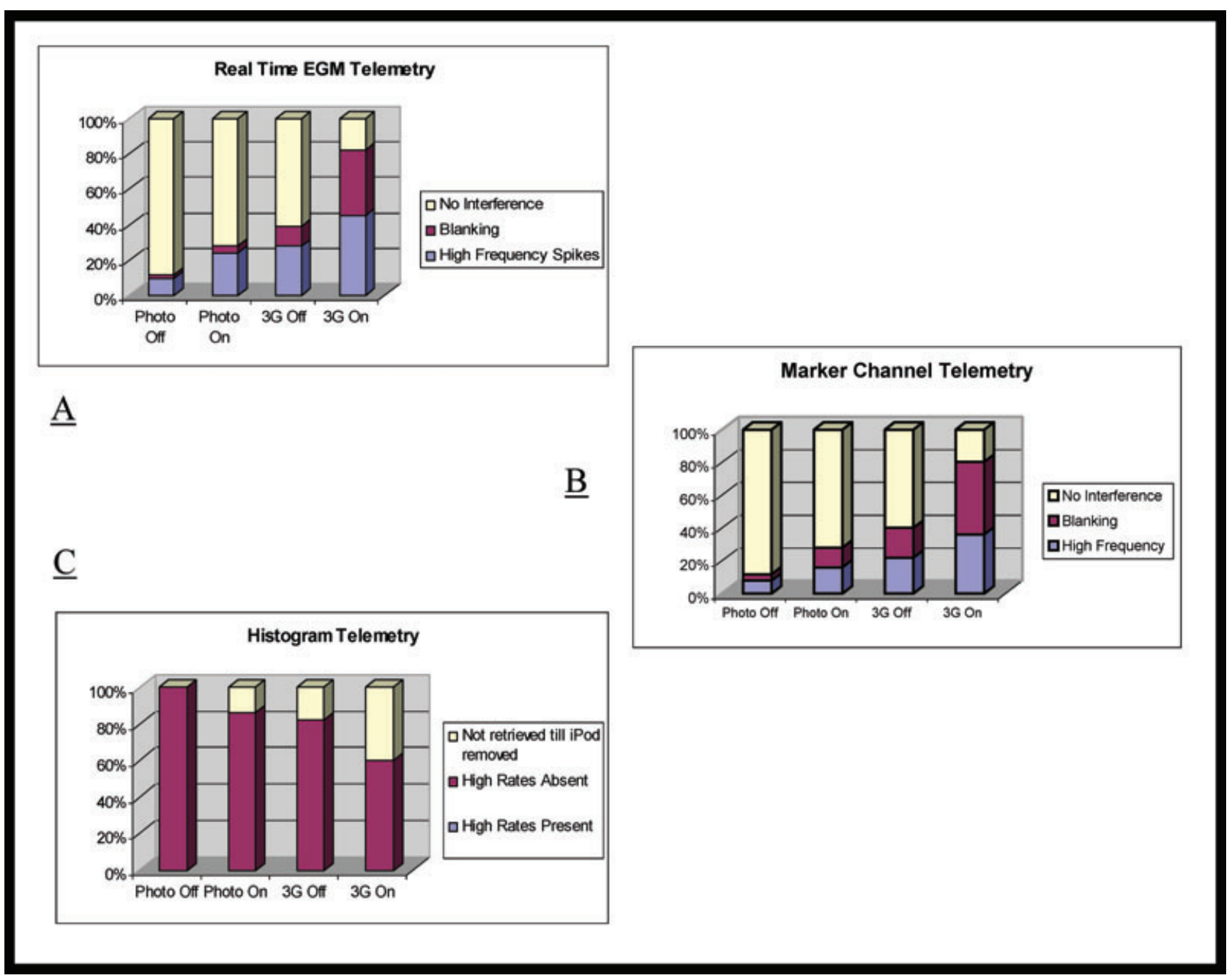

Figure 1. Uplink telemetry data results: (A) real-time EGMs, (B) marker channel, and (C) Histogram. Inductive telemetry uplinking experiences more interference from the iPods, when they were switched "on" compared with when they were "off." Third-generation iPod generated more interference than the Photo iPod. 

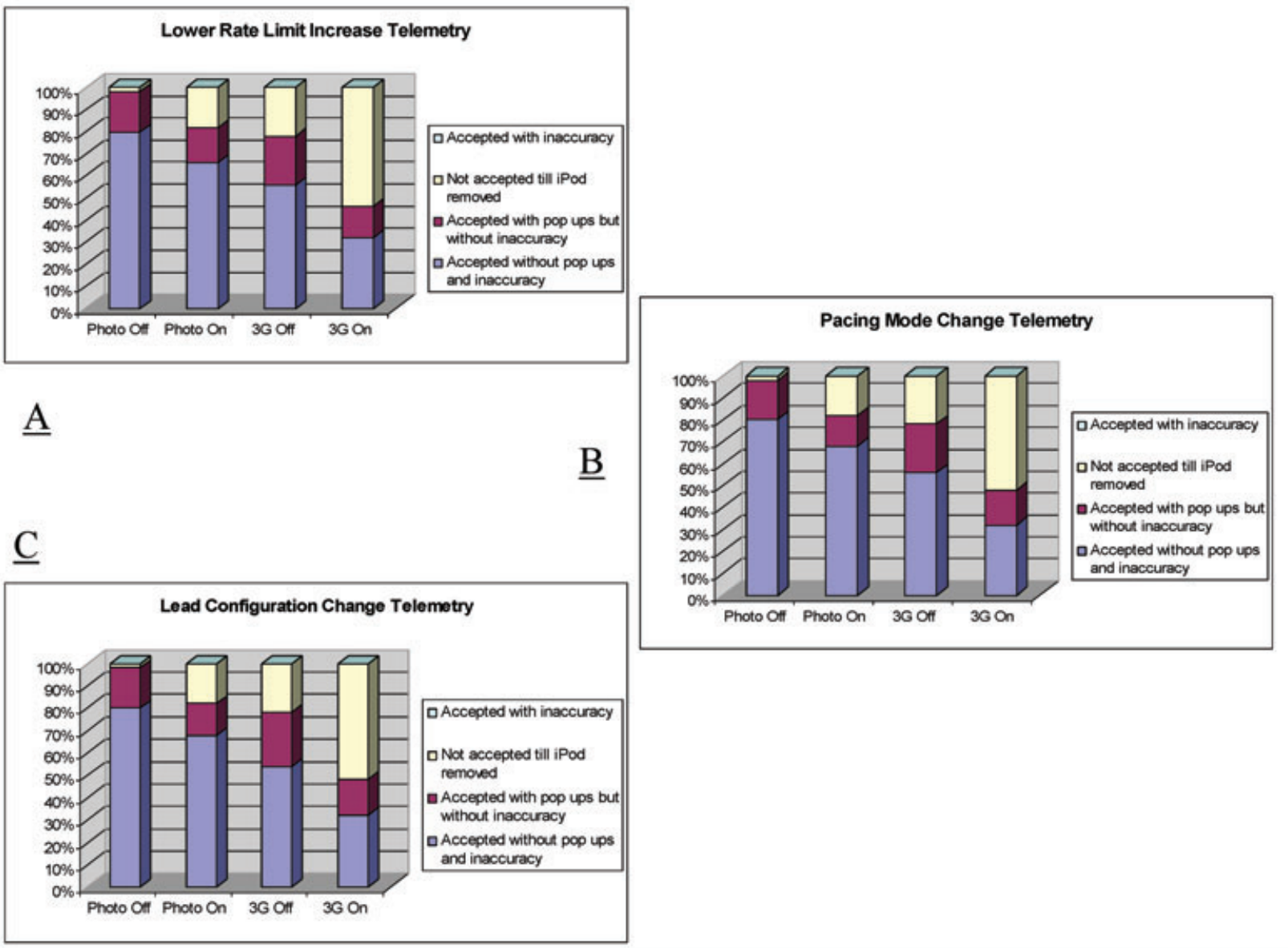

Figure 2. Downlink telemetry data results: (A) lower rate limit increase, (B) pacing mode change, and (C) lead configuration change. Inductive telemetry downlinking experienced interference from the iPods even when the iPod was not "on." However, the interference increased with iPods in "on" state; $3 G$ iPod generated more interference than the Photo iPod. No transmitted data were inaccurately transmitted.

Sequential occurrence of high-frequency spikes and blanking was also observed $(1.4 \%$ tests, Fig. 3A). TI occurred in $25.6 \%$ of patients when the iPod was "on" and in $13 \%$ even with the iPod was turned "off." There were no inaccuracies in downlinked data when the downlink communication was successful.

\section{Clinical Observations}

TI with uplink and downlink transmissions was observed more frequently with $3 \mathrm{G}$ than with photo iPod. A low level of TI was present even when the iPods were turned off, and it increased significantly with iPods in the "on" state (Figs. 1 and 2).

The data transmission from the programmer to the pacemaker (downlink) was normal in the majority of patients. When the data did not downlink at normal speed, these were either delayed or eventually failed. Failure of downlink transmission was accompanied by warning of possible data corruption and/or suggestions to cancel telemetry (Fig. 3B). Data that were downlinked successfully were never corrupted or inaccurate.

\section{Magnetic Field Emissions}

Both models of iPods generate MFE (maximum-0.05 micro Tesla $[\mu \mathrm{T}]$ ) in the range of $30-300 \mathrm{kHz}$, the low-frequency (LF) range (Fig. 4). Worst-case MFE from the wands varied from 0.05 to $0.41 \mu \mathrm{T}$, whereas those from the pacemaker ranged between 0.0001 and $0.0041 \mu \mathrm{T}$ (Fig. 5). The results are displayed in Table I.

\section{Discussion}

Unidirectional telemetry using static magnetic field coupling was superseded by 


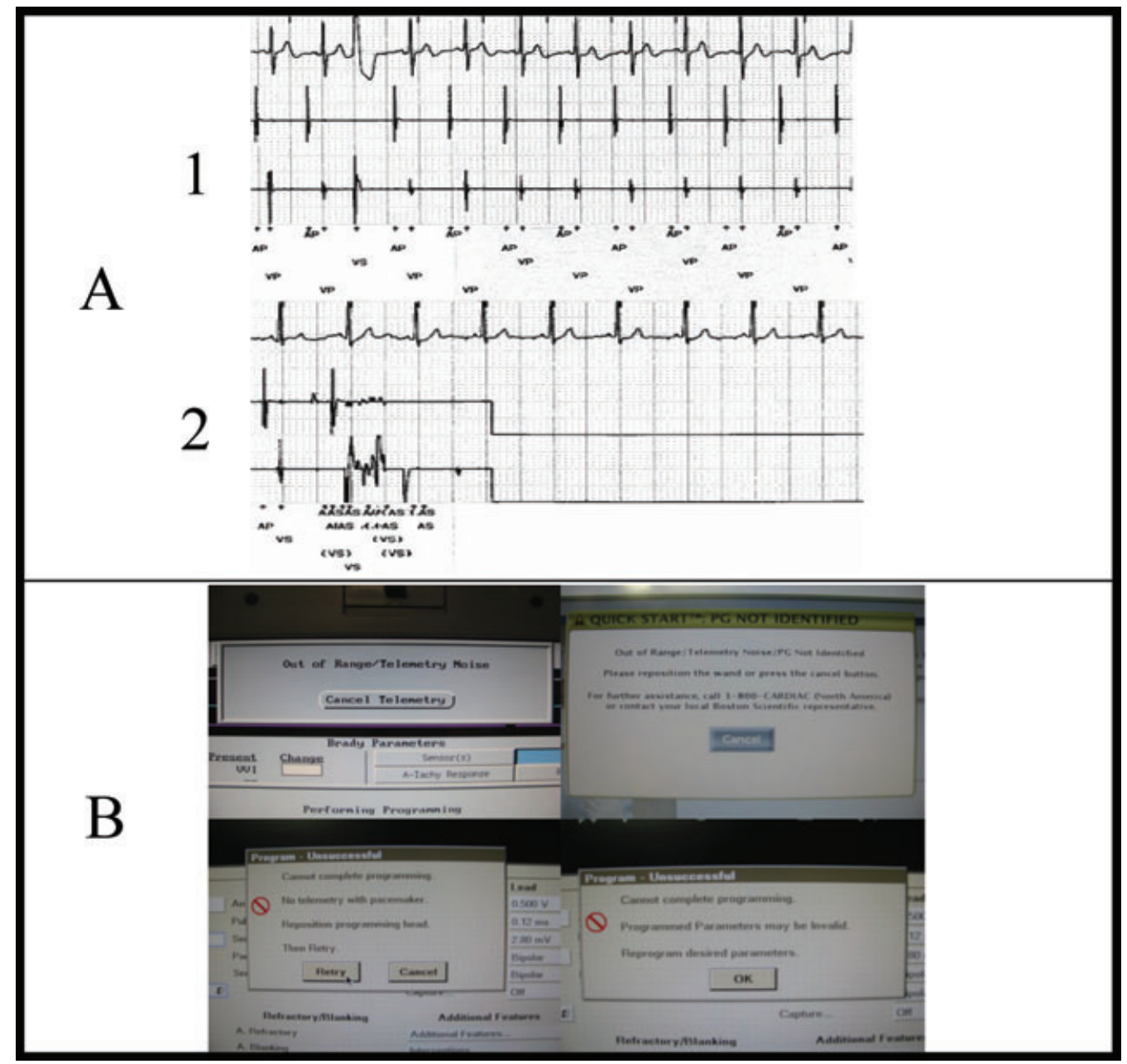

Figure 3. (A) 1-Real-time EGM channels and marker channel recording without interference. 2-High-frequency spikes in the middle and lower channels, followed by complete blanking of these channels suggestive of uplink telemetry interference. Note the corresponding annotations on the marker channel. (Upper channel: surface ECG [lead II]; middle channel: real time atrial EGM; lower channel: real time ventricular EGM; bottom channel: marker channel; AP_atrial paced event, VP-ventricular paced event, AS-atrial sensed event, VS-ventricular sensed event). (B) Four different types of "Pop ups" are displayed on the programmers' (Model 3120, Boston Scientific, and Model 2,090 W, Medtronic) screen suggestive of downlink telemetry interference. They display the messages of failure to establish link between the device and the programmer, failure to maintain link during data transmission, and warning toward possible corruption of downlinked data.

bidirectional telemetry in 1979, two decades after the first pacemaker implantation. Since then, several techniques have been used for telemetry communication, including reflected impedance coupling and RF coupling. ${ }^{9}$ Wanded telemetry, a type of inductive-RF coupling, is the most commonly used method for bidirectional telemetry communication in IHRDs. We studied IT as the first step toward characterizing telemetry interference with IHRDs.

\section{Inductive Telemetry Interference from iPods}

A few characteristics of iPods are responsible for causing the telemetry interference observed in this study. Unintentional MFE from both iPods occur in the low-frequency band employed for IT. Furthermore, the magnitudes of the emissions from the iPod are lower than those employed by the interrogating wand, but are on the same order of magnitude as those from the pacemaker. The emissions are weaker but do not disappear 


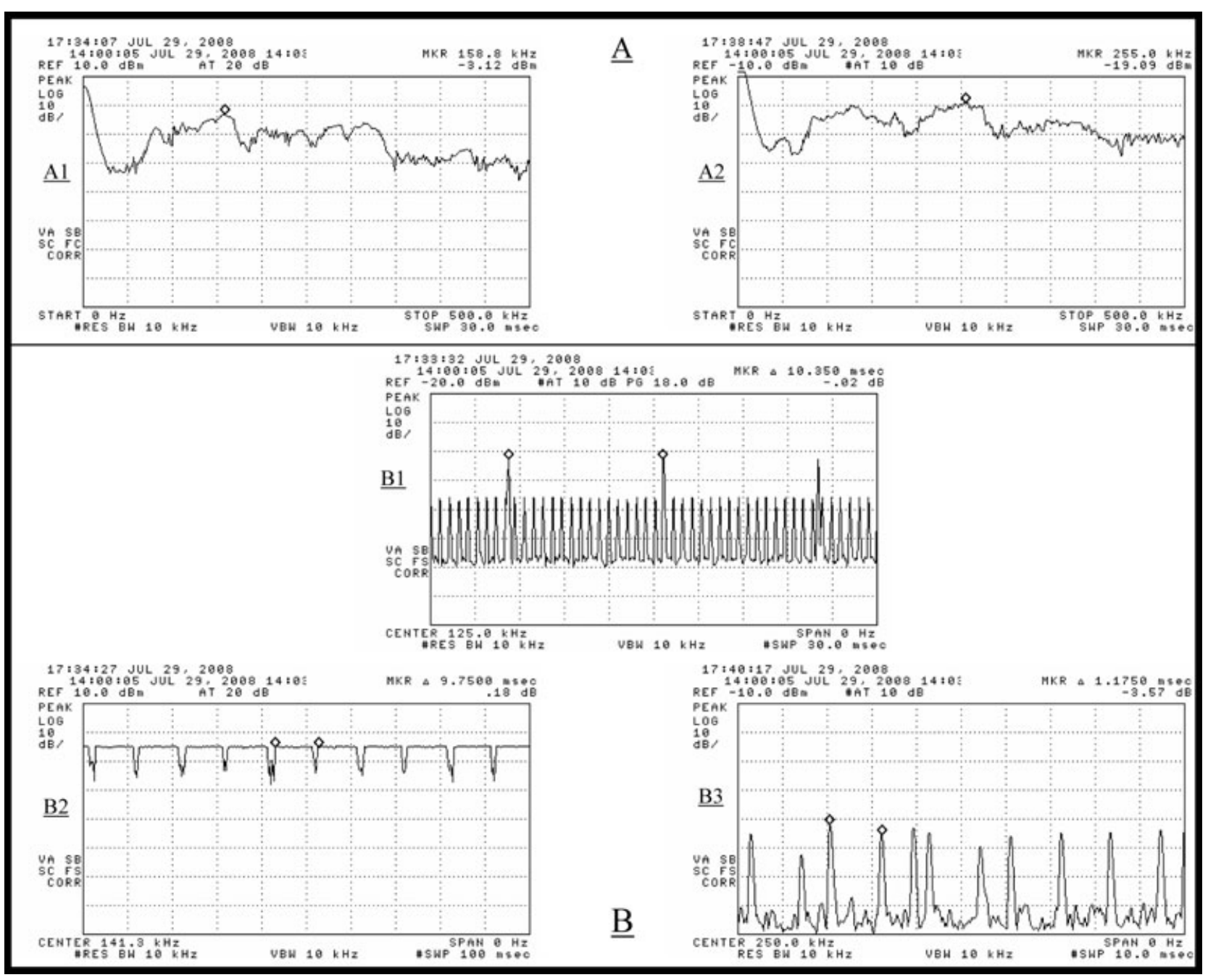

Figure 4. Electromagnetic emissions from $3 G$ and Photo iPods in the "on" and "off" states in the 0-500-kHz range are shown. Both models of iPods generate magnetic field emissions (maximum-0.05 micro Tesla [ $\mu$ T]) in the lowfrequency range (30-300 kHz). (A)—relative magnetic field emissions from iPod up to $500 \mathrm{kHz}$, (A1) $3 G$ turned on with music playing, (A2) Photo turned on with music playing. The reference level is $20 \mathrm{~dB}$ lower than in plot (A1). (B)_Comparison of "on" and "off" states in time domain. (B1) 3G "off" state (keypad sense operating), (B2) $3 G$ on State (music playing), (B3) Photo "off" state. Periodic emission from internal circuitry may be from power circuitry or keypad sensing circuitry. Different modulations can result in different interference based on device mode.

when iPods are turned off. MFE from 3G are 10 to 40 times stronger than those from photo iPod (Table I). Consistent with these features of the media players, we observed more frequent TI with iPods turned on than off and also more frequently with 3G than with photo iPod (Figs. 1 and 2). Other investigators have shown that up to $100-\mathrm{kHz}$ emissions from the iPods generate a localized, less than $2 \mu \mathrm{T}(<1.6 \mathrm{~A} / \mathrm{m})$ magnetic field that is strongest within $1 \mathrm{~cm}$ of their internal hard drives. ${ }^{10}$ These emissions are too weak to significantly couple to the leads or header ends of the pacemaker. Moreover, a pacemaker's signal detection and processing circuitry is protected by low-pass filters and extremely low (frequency) pass filters further downstream. Thus, significant electromagnetic interference is prevented. As iPod emissions are in the working range as the wand, they can still generate TI without altering the pacemaker's pacingsensing functions. Headphones were not used in our study as magnetic flux density of the earbuds alone has been found to be about 100,000 times that of the emissions from iPods. It imparts headphones the capacity to generate clinically significant static magnetic interference, whereas iPods generate only TI. ${ }^{11}$

This study revealed that contemporary devices can differentiate between more and less 


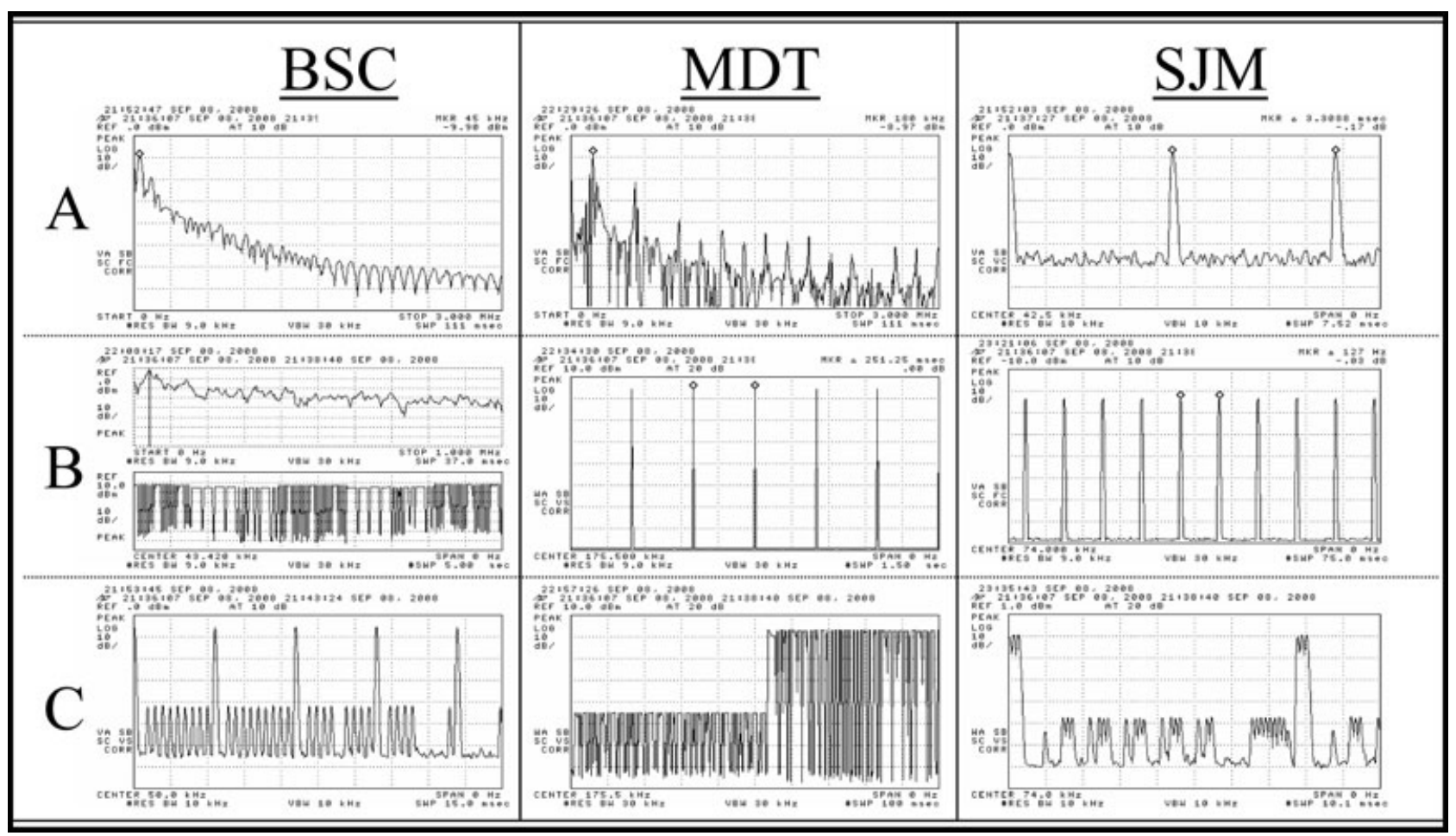

Figure 5. Magnetic field emissions from the wands (A) for the three manufacturers as well as the frequency spectrum of uplink (B) and downlink (C) telemetry communication from the pacemakers are shown. MFE for the wands varied from 0.05 to $0.41 \mu \mathrm{T}$, whereas those from the pacemaker ranged between 0.0001 and $0.0041 \mu \mathrm{T}$. MFE from the wand were much stronger than the emissions from the pacemakers. A-Pacemaker Wand Emission Spectrum is shown. Operating frequencies are $43.4 \mathrm{kHz}$ for BSC, $175 \mathrm{kHz}$ for MDT, and $74 \mathrm{kHz}$ for SJM. B-Uplink transmission of real time data is shown to occur continuously once the link is established. C-During downlink telemetry communication between wand and pacemaker, high pulses are representative of emissions from wand and low pulses are representative of response from pacemaker. BSC $=$ Boston Scientific; $M D T=$ Medtronic; SJM = St. Jude Medical, Inc.

important data for transmission. Downlink data (e.g., upper rate limit, maximum sensor rate, sensor-driven pacing function, voltage and pulse width outputs, sensitivity settings, atrioventricular delay, refractory and blanking periods, mode switch parameters, etc.) are more important than uplink data (e.g., device identification data, battery data, atrial and ventricular thresholds and sensing amplitudes, pacing lead impedance, mode switch episodes, etc.). Corrupted downlink signals could alter pacemaker function and result in dire clinical consequences. Some of the programmers we tested perceived the signals from the iPods as potentially capable of corrupting the intended downlink data. This was manifested by a pop-up suggesting cancellation of downlink telemetry and warning for the possibility of data corruption (Fig. 3B). The warning persisted as long as the iPod was near the wand. However, we did not observe any corruption of successfully downlinked data. Additionally, stored uplink data are regarded to be more important than the real time uplink data. It is important that stored data like battery life, percentage of paced-sensed events, arrhythmia burden, and recorded electrograms are transmitted securely as they constitute vital information for clinical decision making. Real-time uplink data are most prone to telemetry interference. This should be borne in mind at the time of pacemaker interrogation and programming.

Establishment of the telemetry link between the device and its programmer is an important first step, followed by the maintenance of the link and data transfer. ${ }^{12}$ Emissions from iPods prevent the establishment of the link (Fig. 3B). Maintenance of the link is based on continuous "handshake" and iPods interfere with it by causing sudden delinking, which is manifested by blanking of real-time data transmission on the programmer screen. Though blanking is not a phenomenon specific to TI, it persisted until the interfering emissions disappeared. During IT, downlinking of vital data normally triggers blanking. It is manifested as a transient and self-reverting cessation of real time uplink data transmission. Considered as normal, it is suggestive of slow sequential functionality of IT, 
Table I.

Magnetic Field Emissions

iPod Testing (Worst-Case Scenario)

\begin{tabular}{lccccc}
\hline & \multicolumn{2}{c}{ On } & & \multicolumn{2}{c}{ Off } \\
\cline { 2 - 3 } \cline { 5 - 6 } Device & H-field $(\mathbf{A} / \mathbf{m})$ & B-field $(\mu \mathbf{T})$ & & H-field $(\mathbf{A} / \mathbf{m})$ & B-field $(\mu \mathbf{T})$ \\
\hline iPod 3G (SN:JQ4036S9QQF) & 0.04229 & 0.05314 & & 0.01276 & 0.01604 \\
iPod Photo (SN:Q509NE1SAY) & 0.00426 & 0.00536 & & 0.00038 & 0.00048
\end{tabular}

Wand and Pacemaker Testing

\begin{tabular}{|c|c|c|c|c|c|}
\hline \multirow[b]{2}{*}{ Manufacturer } & \multirow[b]{2}{*}{ Operating Frequency (kHz) } & \multicolumn{2}{|c|}{ Wand Interrogation } & \multicolumn{2}{|c|}{ Pacemaker Response } \\
\hline & & H-field (A/m) & B-field $(\mu \mathrm{T})$ & H-field (A/m) & B-field $(\mu T)$ \\
\hline Boston Scientific & 43.4 & 0.30208 & 0.37960 & 0.00011 & 0.00013 \\
\hline Medtronic & 175.0 & 0.32328 & 0.40624 & 0.00323 & 0.00406 \\
\hline St. Jude Medical & 74.0 & 0.04399 & 0.05528 & 0.00247 & 0.00311 \\
\hline
\end{tabular}

( $\mathrm{H}$-field = Magnetic field strength measured in Amperes/meter $(\mathrm{A} / \mathrm{m})$, B-field = Magnetic flux density measured in microTesla $(\mu \mathrm{T})$. These measurements are mutually related, interconvertible and bear similar clinical importance. $\mathrm{B}=\mu \mathrm{H}$, where $\mu$ is the magnetic permeability of the medium.)

which can be attributed to limited bandwidth of LF emissions (Fig. 5C). The LF emissions reduce the speed of data transmission to usually $2-5 \mathrm{kbps}$, forbidding simultaneous bidirectional transmission. Perennial flow of messages checking validity of to and from link interspersed with more important security checks helps to keep the transmission safe and accurate. This process comprises the majority of time during data transmission. With new devices utilizing radio emissions from ultra highfrequency ( $300 \mathrm{MHz}-3 \mathrm{GHz}$ ) range, data transmission rate is increased by more than $\sim 10$ times of that with wanded devices. RF telemetry imparts complex multi-functionality and capability of simultaneous bidirectional transmission. ${ }^{13}$

\section{Study Limitations}

This is a relatively small observational study on inductive telemetry interference from only one source (iPods). Characteristics of interference seen with IT may not be extrapolated to remote RF telemetry, which is increasingly being used in implantable defibrillators, cardiac resynchronization devices, and more recently, in pacemakers. Because of theoretical interference that could be observed with remote monitoring systems, additional research is needed.

\footnotetext{
References

1. Thaker JP, Patel MB, Jongnarangsin K, Liepa VV, Thakur RK. Electromagnetic interference with pacemakers caused by portable media players. Heart Rhythm 2008; 5:538-544.

2. Webster G, Jordao L, Martuscello M, Mahajan T, Alexander ME,
}

\section{Clinical Implications}

Understanding TI elucidates the potential vulnerabilities and mechanisms by which device integrity may be challenged through improper data transmission, leading to corruption of programmed parameters. Within the boundaries set by the Federal Communications Commission, medical implants are acquiring more advanced remote wireless communication capability at higher RFs than that used previously. However, as the devices become more sophisticated, they achieve more data acquisition and storage capability, accuracy, and speed of communication. Whether domestic devices including iPods can cause significant TI with wireless RF and inductive telemetric communication with RMS remains to be tested.

\section{Conclusions}

iPods do not cause pacemaker malfunction because of the low power of their magnetic field emissions and continuous parity check protocols used by pacemakers during communication. However, the emissions from iPods can produce interference with establishment and maintenance of a telemetry communication link and transmission of real time data.

Cecchin F, Triedman JK, et al. Digital music players cause interference with interrogation telemetry for pacemakers and implantable cardioverter-defibrillators without affecting device function. Heart Rhythm 2008; 5:545-550. 
SHAH, ET AL.

3. Thaker JP, Patel MB, Jongnarangsin K, Liepa VV, Castellani M, Thakur RK. Electromagnetic interference in an implantable loop recorder caused by a portable digital media player. Pacing Clin Electrophysiol 2008; 31:1345-1347.

4. Savci H, Sula A, Wang Z, Dogan NS. MICS Transceivers: Regulatory standards and applications (Medical Implant Communications Service). Proc IEEE Southeast Con 2005; 179182.

5. Seidman SJ, Rugggera PS, Brockman RG, Lewis B, Shein MJ. Electromagnetic compatibility of pacemakers and implantable cardiac defibrillators exposed to RFID readers. Intern J Radio Freq Ident Technol Appl 2007; 1:237-246.

6. Van Der Togt R, van Lieshout EJ, Hensbrock R, Beinat E, Binnekade JM, Bakker PJM. Electromagnetic interference from radio frequency identification inducing potentially hazardous incidents in critical care medical equipment. JAMA 2008; 299:28842890 .
7. Berwick DM. Taming the technology beast. JAMA 2008; 299:28982899.

8. Halperin D, Clark SS, Fu K, Heydt-Benjamin TS, Defend B, Kohno T, Ransford B, et al. Pacemakers and implantable cardiac defibrillators: Software radio attacks and zero-power defense. Proc IEEE 2008 129-142.

9. Duru F, Lauber P, Klaus G, Reto C. Hospital pager systems may cause interference with pacemaker telemetry. Pacing Clin Electrophysiol 1998; 21:2353-2359.

10. Bassen H. Low frequency magnetic emissions and resulting induced voltages in a pacemaker by iPod portable music players. BioMedical Engineering OnLine 2008; 7:7.

11. Lee S, Ransford B, Fu K, Kohno T, Maisel WH. Electromagnetic interference (EMI) of implanted cardiac devices by MP3 player headphones. Circulation 2008; 118:596.

12. Grevious J. US Patent 6167310 Dec. 26, 2000.

13. FCC website at http://wireless.fcc.gov 